\title{
How does caffeine affect the athlete's body?
}

\author{
Nadezhda Shuvalova ${ }^{1 *}$, Svetlana Lezhenina ${ }^{2}$, Venera Akimova ${ }^{2}$, Marina Grigorieva ${ }^{2}$, Sergey Zorin ${ }^{1}$ \\ ${ }^{1}$ Chuvash State Pedagogical University Named After I. Ya. Yakovlev, 428000, Cheboksary, Russia \\ ${ }^{2}$ Chuvash State University Named After I. N. Ulyanov, 428015, Cheboksary, Russia
}

\begin{abstract}
The purpose of the research is to carry out a theoretical analysis and generalization of modern scientific and methodological literature on the problem of the caffeine impact intake on the body of athletes. The results of experimental researches indicate a positive effect of caffeine intake on the indices of the anaerobic tests results and on the speed-strength indices. It has also been found that caffeine in combination with creatine enhances each other's actions and has a positive effect on endurance performance. When evaluating the effect of caffeine on the athlete's body, it was found that caffeine affects the production of lactic acid. It turned out that lactic acid is formed even at rest, as a side effect of taking caffeine. It is found that caffeine inhibits the activity of the enzyme phosphodiesterase. It was found that caffeine increases the secretion of endomorphins and, due to this, has an analgesic effect.
\end{abstract}

\section{Introduction}

The features of caffeine effects on the body of athletes have been studied relatively recently. The point of caffeine action is the human central nervous system. The mechanism of action is to stimulate its functioning with caffeine [1,2]. Adenosine and dopamine regulate biochemical processes in the brain. Adenosine weakens the processes of excitation in the brain and, thus, protects it from overwork. In terms of their chemical structure, the molecule of adenosine and caffeine is almost identical. Due to this, caffeine can competitively replace it at the brain receptors. Therefore, caffeine can block the action of adenosine. And thus have a stimulating effect on the central nervous system. Caffeine is also capable of providing a normotimal effect, increasing the level of psychological stability and mental performance $[3,4]$.

Because of this, the popularity of caffeine as an ergogenic support has increased in recent years. [5.6]

New dietary supplements containing caffeine have emerged in the form of "sports drinks," "energy drinks," and so on. Attempts to take advantage of the promoting athletic performance benefits are generating demand for this type of sports supplement.

Caffeine is a psychoactive substance that can cause drug dependence [7]. Most of the people who regularly consume caffeine-containing beverages are unable to reduce their intake, even with health problems. It is believed that regular consumption of more than $450 \mathrm{mg}$ of caffeine per day increases the risk of cardiovascular disease, neurological disorders, and osteoporosis. [8.9]

We are well aware of caffeine effect on the body's aerobic capacity, but the mechanisms of action are not yet fully understood. If there is information about the changes that caffeine can cause, it is only in sports with manifestations of aerobic endurance (marathon; race walking, etc.). The changes that occur during predominantly anaerobic or mixed exercise are still being studied in detail. There are already enough experimental studies, but the results are often contradictory. It may be due to the different design of the experiments, with different types of subjects (persons related to professional sports, amateurs or untrained people).

Therefore, the purpose of our research was to carry out a theoretical analysis and generalization of modern scientific and methodological literature on the problem of caffeine effect intake on the body of athletes.

\section{Materials and methods}

\footnotetext{
* Corresponding author: msta77@yandex.ru
} 
To achieve the goal of the research, we carried out an abstract review of domestic and foreign literary sources, which presented the results of experimental studies of the features of caffeine effect on the indicators of anaerobic and aerobic performance of the body, the manifestation of speed, speed-power and strength abilities, physical performance, general endurance of individuals, practicing and not going in for sports.

As the main research method we used the methods of theoretical analysis and generalization of literature data.

\section{Results and discussion}

The test most commonly used to assess anaerobic performance is the Wingate Anaerobic Test. The athlete must perform on the ergometer for 30 seconds, depending on the speed of rotation, the load, taking into account his body weight.

Medbo performed the Wingate anaerobic test three times. This resulted in energy consumption ranging from 60 to $84 \%$ of ATP production regardless of oxygen supply. However, no statistically significant difference was found between the caffeine and non-caffeine groups [10].

In Kang's experiment [11] with the participation of professional cyclists and untrained people, indicators of an increase in the total amount of energy released were recorded. The subjects used caffeine doses of 2.5 and 5 $\mathrm{mg} / \mathrm{kg}$, respectively. While this test is considered representative, it does not take into account the total energy expenditure of some more interval type exercise sports such as football, ice hockey, etc. The increase in total energy released was positive when athletes received caffeine at a dose of $6 \mathrm{mg} / \mathrm{kg}$ in the experiment.

Contrary to these findings, Williams [12] found no change after 15 seconds of exercise, but rather found a beneficial effect after short 6-7 seconds of exercise.

\section{Effect of caffeine on speed performance}

Paton [13] studied the effects of caffeine in 20 athletes. They mainly trained in athletics sports (sprint distances). In the experiment, all athletes used a standard set of exercises: 10 accelerations of $20 \mathrm{~m}$ with a break of 10 seconds between separate repetitions. All athletes received caffeine at a dose of $6 \mathrm{mg} / \mathrm{kg}$, the control group received a placebo. In the first acceleration, the group of athletes who took caffeine showed a significant advantage in the speed of running the segment, compared to the results of the athletes in the control group. The running time of the last 10th acceleration increased by $14 \%$ in the "Placebo" group and by $14.7 \%$ in the "Caffeine" group.

Stewart's experiments [14] gave slightly different results. He studied the effects of caffeine on rugby players over 7 simulated matches, ranging from 2 to 40 minutes, with a 10-minute break between halves, where the players received caffeine at a dose of $6 \mathrm{mg} / \mathrm{kg}$. Caffeine increased the efficiency of anaerobic performance by an average of $0.5-3.4 \%$.

The results of a similar study involving swimmers were similar: trained athletes and untrained athletes swam 2 times $100 \mathrm{~m}$ with a break of 10 minutes. Swimming results for the second leg after taking caffeine were significantly higher than the swimming time for the first leg. At the same time, the effect of faster overcoming the distance after taking caffeine was higher in trained athletes.

The essence of the experiment, aimed at studying the effect of caffeine on the exercise of endurance, was to measure the maximum accumulated oxygen deficiency after exercise lasting from 1 to 3 minutes. This load corresponds to an over-maximum intensity of $125 \%$ VO2max.

The accumulation of oxygen deficiency was not observed, and thus the time to complete the exercise on bicycle ergometry increased after taking caffeine by $14 \%$. It proves the positive effect of caffeine on endurance. In another experiment, the subject was loaded for maximum of 2 minutes on a bicycle ergometer and immediately after that at speed corresponding to run of $800 \mathrm{~m}$. The result in the form of energy expenditure was $794 \pm 164 \mathrm{~W}$ higher with the introduction of caffeine [15].

Other authors [16] administered caffeine in combination with creatine and found a synergistic positive effect of their combined use.

In resistance training, the intensity of the load is calculated as a percentage of the amount of weight (in $\mathrm{kg}$ ) that the athlete can overcome with a single repetition. Therefore, the maximum is a load that can only be done once and is expressed as $100 \%$.

Green [17] tested 17 subjects who performed 3 exercises on bench press and leg press to $80 \%$ of peak power in a double-blind research using caffeine at $6 \mathrm{mg} / \mathrm{kg}$ and placebo. The first two attempts showed no difference, only the third was positive.

Hudson et al. [18] in tests of the legs and arms muscles strength, as in the model of resistance exercise to induce fatigue, found significantly higher repetitions of the exercise after administration of caffeine. The authors emphasized the need to take into account athletes' coffee addiction.

Beck [19] conducted an experiment with two groups of randomly selected people performing a set of exercises: bench press and leg press at $80 \%$ peak power until fatigue. The caffeine group performed better on the bench press $(34 \mathrm{~kg}$ ) than the placebo group $(24 \mathrm{~kg})$. At the same time, there were no significant differences 
in the leg press results. However, other experiments by other authors have not confirmed these results. In particular, the positive effect of caffeine intake on the manifestation of the lower extremities muscles strength capabilities was found [20].

This discrepancy, which was later discovered by other authors, is difficult to explain. One of the reasons may be that the experiments were conducted with people who had no experience with resistance training.

With an increase in adrenaline, lactic acid accumulates. Some researches have shown that caffeine intake can interfere with lactic acid production even at rest as a side effect. However, there is no ergogenic effect in these cases. The described effect of caffeine was manifested only in a few subjects. This discrepancy has led to the suggestion that the caffeine-lactic acid causal relationship is not direct, and there are other explanations.

Caffeine causes an increased production of adrenaline by the adrenal glands. Both epinephrine and norepinephrine release more glucose into the bloodstream at the start of a workout. Studies showing a positive effect were conducted with trained individuals, while untrained subjects did not have it.

Caffeine has been shown to increase irritability and contractility by stimulating $\mathrm{Na}+/ \mathrm{K}+$ ATPase activity.

Some authors explain this by a decrease in plasma $\mathrm{K}+$ concentration. During muscle contraction, depolarization of muscle cells occurs, accompanied by the outflow of $\mathrm{K}+$ into the extracellular fluid, from which it diffuses into the blood. This relieves fatigue and creates more favorable conditions for further muscle contractions. Caffeine metabolites also favorably inhibit this interaction by stimulating $\mathrm{K}+$ transport. Caffeine increases plasma $\mathrm{K}+$ during aerobic exercise.

When analyzing the literature data, it was found that caffeine inhibits the activity of the enzyme phosphodiesterase. It has also been shown that caffeine increases calcium mobilization in the sarcoplasmic reticulum. It may have a beneficial effect on muscle fiber contraction.

It is believed that the perception of muscle pain decreases when they act as agonists, and, conversely, increases when they act as antagonists. This results in a decrease in maximum contact (MVC) and travel speed. Caffeine is used as a common component of pain relievers for its properties, i.e. by blocking adenosine receptors. It is often combined with paracetamol to increase its effectiveness.

The use of caffeine for pain suggests that, in addition to the mentioned effect on adenosine, it is involved in the regulation of exercise by increasing the involvement of motor muscle units. If caffeine reduces pain, it allows more units to be recruited and thus increases muscle strength. This theory will explain the effects of caffeine even with anaerobic exercise [21]. However, its effect could not be demonstrated on EMG recordings.

Some differences have been found between men and women in the perception of pain [22]. A reduced doseresponse was observed with a $10 \mathrm{mg} / \mathrm{kg}$ dose of caffeine versus a $5 \mathrm{mg} / \mathrm{kg}$ dose in men after 30 minutes on a bicycle ergometer at $60 \%$ VO2max. No similar dose-response results were found in women, and a decrease in pain threshold was observed at all doses. It is believed that women have a higher threshold for sensitivity to pain.

In addition, the analgesic effect of caffeine can be attributed to the fact that it increases the secretion of endomorphins. These are chemical compounds that are produced in the tissues of the brain and they can reduce pain and positively affect the emotional state of a person. Endomorphins are synthesized in large quantities in the human body during sports competitions. This makes it possible to ignore the onset of pain feeling and to mobilize due to this additional physical and mental reserves of the body.

Since caffeine reduces pain during prolonged exercise, it is likely that this effect also applies to the perceived effort method. Doherty [23] found that the sensitivity decreased by $5.6 \%$ compared with placebo. The same author found that the fall is 1 point on the Borg scale [24] with an intense load on the ergometer.

The effect of caffeine has rarely been tested during maintenance exercise, although it would help significantly in evaluating effort. Green [25] found no difference between the caffeine and placebo groups, but found an increase in exercise repetitions. Perhaps this is one of the first positive results in exercises with a predominantly anaerobic energy supply mechanism.

Caffeine has also been studied as a means of reducing fatigue. However, it must be borne in mind that there is central and peripheral fatigue. When you measure fatigue during aerobic exercise, it is clear that caffeine prolongs and increases performance. [26] However, there is also evidence that it also has a positive effect on speed-strength performance, but this effect is difficult to prove. After analyzing the literature data, we came to the conclusion that caffeine can significantly increase the time before the onset of fatigue and, thanks to this, maintain the strength of muscle contractions during muscular loads close to maximum.

\section{Conclusions}

An abstract review of literary sources allows us to conclude that caffeine has a positive effect on the indicators of the body anaerobic capabilities and speed-strength capabilities of athletes.

The mechanism of caffeine influence on increasing the endurance of athletes is a positive effect on the increase in the secretion of endomorphins - substances produced in the tissues of the central nervous system and having the ability to reduce pain and positively influence the emotional profile of athletes. 
Thus, caffeine is found in many energy drinks that athletes consume. To achieve a positive effect of their intake on the body and motor abilities, every coach and athlete must know about the patterns and mechanisms of their impact.

\section{References}

1. J.I. Medbo, I. Tabata, J Appl Physiol, 67, 1881-1886 (1989)

2. H. Kang, H. Kim, B. Kim, J Exerc Physiol Online, 1(3) (1998)

3. K.T. Schneiker, D. Bishop, B. Dawson, Med Sci Sports Exerc, 38, 578-585 (2006)

4. J.H. Williams, J.F. Signorie, J.S. Barnes, Br Sports Med, 22, 132-134 (1988)

5. C.D. Paton, W.G. Hopkins, L. Vollebreght, Med Sci Sports Exerc, 33, 822-825 (2001)

6. G.R. Stuart,W.G. Hopkins, Med Sci Sports Exerc, 37, 1998-2005 (2005)

7. M. Roberty, P.M. Smith, Scand Med Sci Sports, 15, 69-78 (2005)

8. P. Hespel, B. Op't Ejinde, M. Van Leemputte, J Appl Physiol, 92, 513-518 (2002)

9. J.M. Green, J.P. Wickwire, J.R. McLester, Int J Sport Phys Per, 2, 250-259 (2007)

10. G.M. Hudson, J.M. Green, Med Sci Sports Exerc, 39, S248 (2007)

11. T.W. Beck, I. Jacobs, J.R. Schmidt, J Srength Cond Res, 20, 506-510 (2006)

12. T.A. Astorino, R.L. Rohmann, K. Firth, Eur J Appl Physiol, 102, 127-132 (2008)

13. K.J. Davis, J.M. Green, Sport Med, 39, 813-832 (2009)

14. F. Greer, C. McLean, J Appl Physiol, 85, 1502-1508 (1998)

15. M.J. Crove, A.S. Leicht, W.L. Spinks, Int J Sports Nutrition Exerc Metab, 16, 528-544 (2006)

16. R.A. Barraco, V.L. Coffin, Brain Res, 272, 392-395 (1983)

17. J.M. Kalmar, Med Sci Sports Exerc, 12, 2113-2119 (2005)

18. R.W. Motl, P.J. O' Connor, Med Sci Sports Exerc, 38, 598-604 (2006)

19. M. Doherty, P.M. Smith, R.C.R. Davidson, et al. Med Sci Sports Exerc, 34, 1785-1792 (2002)

20. M. Górnicka, J. Pierzynowska, E. Kaniewska, K. Kossa-kowska, A. Woźniak, Rocz Panstw Zakl Hig, 65(2), 113-7 (2014)

21. D.C. Mitchell, C.A. Knight, J. Hockenberry, R. Teplan- sky, T.J. Hartman, Food Chem Toxicol, 63, 136-42 (2014)

22. S.E. Meredith, L.M. Juliano, J.R. Hughes, R.R. Grif-fiths, J Caffeine Res, 3(3), 114-130 (2013)

23. O.E. Zaitseva, A.L. Frolov, F.M. Bayramgulov, F.S. Zarudiy, R.T. Rakhmanova, N.I. Shigaev, A.F. Maksyutova, G.F. Salikhova, Pharmacogenetics of alcohol, caffeine, nicotine in pregnant women and perinatal risks: guidelines of the Ministry of Health of the Republic of Belarus (Ufa, 2013)

24. A.S. Kuznetsova, Z.M. Kuznetsova, Russian Journal of Physical Education and Sport, 14(4), 5-7 (2019)

25. S.V. Shterman, Sports nutrition products (AP "Stolitsa", M., 2017) 